\title{
Adaptación de la Escala de Percepción de Restauración Ambiental en parques
}

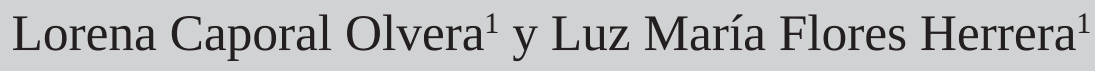

\section{Introducción}

La evaluación de la restauración psicológica requiere instrumentos con las propiedades psicométricas correspondientes y aun así es un reto cuando se realiza en ambiente natural. De ahí que un avance crucial en este dominio ha sido dirigir la atención hacia la construcción de escalas basadas en principios psicológicos en contextos naturales (Ulrich, 1983). En este sentido, en los últimos años, se hace evidente la creciente atención dirigida hacia la definición, medición e interpretación del proceso de restauración psicológica. En estudios previos se reconoce la necesidad de adaptar nuevas escalas apropiadas al contexto natural (Hartig, 2003) o de construir instrumentos (Hartig y Staats, 2006). El problema de la medición de la restauración psicológica es que requiere, además del diseño de instrumentos acordes a las particularidades del contexto natural de los sujetos evaluados, identificar su validez ecológica con fines primera-

1 Universidad Nacional Autónoma de México. 
mente evaluativos. Existen estudios realizados en México sobre restauración psicológica, específicamente en escenarios naturales, en los que se adaptaron pruebas para medir el contexto. Un ejemplo de ello es el trabajo de Martínez (2010), quien evaluó el escenario residencial en la restauración psicológica.

El presente estudio financiado por DGAPA-PAPIIT (proyecto IN308811), se propuso adaptar una escala de restauración con las propiedades psicométricas, apropiada a las características de visitantes a jardines. Acorde con los señalamientos de Kaplan y Kaplan (1989), la percepción de restauración psicológica se refiere a que un lugar (construido o natural) es capaz de renovar los recursos atencionales individuales debido a que cuenta con características ambientales no dañinas y como beneficio adicional puede fomentar la reflexión (Fitch, 1983). Es un escenario evaluado como factible para reponer los recursos emocionales y funcionales agotados, contribuye a la reducción del estrés y la fatiga mental (Kaplan, 1995). Por tanto, se puede decir que la restauración psicológica es la renovación de los recursos físicos, psicológicos y sociales ante las demandas adaptativas que provienen de los escenarios sociofísicos (Hartig, 2003). Dada la importancia de esta variable, su evaluación ha sido relevante. Una de las escalas más utilizadas a nivel internacional para evaluar la restauración psicológica es la escala realizada por Hartig y Korpela, con 26 reactivos, de carácter directo, formulados con opciones de respuesta $0=$ Nada y $6=$ completamente, agrupados en cuatro dimensiones: "Estar alejado", "Fascinación", "Coherencia" y "Compatibilidad". Diversos estudios han probado las cualidades psicométricas (Martínez, 2010; Van Den Berg et al., 2003-2007); sin embargo, son escasos los estudios sistemáticos que han evaluado las propiedades psicométricas de la escala en escenarios restauradores como los jardines. En este trabajo se presenta el estudio de las propiedades psicométricas de la escala aludida. 


\section{Método}

Participantes

La muestra estuvo integrada por 200 visitantes jóvenes y adultos de entre 15 y 32 años de edad, 102 hombres (51\%) y 98 mujeres (49\%). Con respecto al estado civil, $90.5 \%$ reportaron ser solteros; en cuanto a la ocupación, en su mayoría resultaron ser estudiantes $(62.5 \%)$.

\section{Material}

La Escala de Percepción de Restauración Ambiental-Revisada (EPRA-R, Martínez, 2010) es unidimensional, consta de 26 reactivos escalares de 11 puntos con opciones de respuesta que van de $0=$ Nada y $10=$ Completamente de acuerdo, con cinco factores: "Compatibilidad" (5 reactivos), "Estar alejado" (5 reactivos), "Fascinación" (5 reactivos), "Alcance" (4 reactivos) y "Coherencia" (4 reactivos).

\section{Procedimiento}

La escala se aplicó dentro de alguno de los cinco parques elegidos para el estudio ubicados en el Distrito Federal, a personas que se encontraban en sus inmediaciones. Se les abordó individualmente, pidiéndoles su participación voluntaria en una investigación realizada para conocer su opinión acerca del parque, garantizando el anonimato. El tiempo aproximado de aplicación fue de 15 minutos y por lo regular los participantes se encontraron sentados. Dentro de la escala se les pidió que eligieran el lugar de más agrado para ellos dentro del parque y que con base en ese lugar de preferencia contestaran las oraciones presentadas. Se dejó claro que ante cualquier duda podían preguntar al encuestador.

La Escala de Percepción de Restauración Ambiental-Revisada fue sometida a una adaptación (por reactivo y por factor) para obtener los análisis adecuados en el escenario utilizado 
en esta investigación. Se realizó un análisis de constructo mediante un Análisis Factorial con Rotación Varimax y un análisis de correlación de Pearson. Asimismo, se obtuvo la consistencia interna de la prueba mediante la prueba Alpha de Cronbach.

\section{Resultados}

Primeramente, para identificar qué reactivos discriminaron entre altos y bajos puntajes se aplicó la prueba t de Student, obteniendo que sólo 17 de los 26 reactivos (de EPRA-R) permitieron discriminar con un nivel de significancia de .000. Es decir, la eficacia de los reactivos elegidos es óptima para medir puntajes de este tipo en la población mexicana. Una vez conociendo que 17 reactivos discriminaban significativamente, se procedió a conocer la estructura factorial.

Con la finalidad de analizar la manera en que se aglutinan los reactivos, se procedió, en primer lugar, a realizar un análisis de componentes principales con rotación varimax, que mostraron adecuados ajustes entre el número de ítems por factor: "estar alejado" (4 reactivos), "coherencia" (3 reactivos), "fascinación" (6 reactivos) y "alcance" (4 reactivos), todos los reactivos mostraron una carga $>.400$. Enseguida, se calculó la varianza explicada por factor, siendo de 54.17\%, 14.20\%, 24.95\% y 7.96\%, respectivamente.

Con el propósito de evaluar la convergencia entre los factores, se realizó un análisis de correlación mediante la prueba de Pearson, se comprobó que los cuatro factores correlacionaron significativamente $(p=.01)$ con una correlación moderada $(r=.40)$; lo que permite establecer que entre ellos se complementan para medir "Percepción de restauración ambiental", con base en estos tres procesos estadísticos se puede indicar que la escala es válida.

Finalmente, se calculó la consistencia interna por factor y total a través del cálculo del coeficiente alfa de Cronbach: por factor "fascinación" $\alpha=.815$; "estar alejado" $\alpha=.678$; "alcance" $\alpha=.695$; y "coherencia" $\alpha=.494$; total $\alpha=.855$, que resultó adecuada, posibilitando señalar que la escala es fiable. 


\section{Discusión}

Según Ulrich (1983), la restauración psicológica se define en términos de una ausencia de estrés $y$, por lo tanto, se ven favorecidos los niveles de respuesta psicofisiológica de las personas. El presente estudio tuvo como propósito evaluar las propiedades psicométricas de la escala de percepción de restauración ambiental revisada de Martínez (2010), adaptada para jardines urbanos. Los resultados han dado cuenta de propiedades similares a dicho instrumento. La escala demostró ajustarse a un modelo unidimensional. Los rubros que cubre: "estar alejado", "coherencia", "fascinación" y "alcance", constituyen cuatro aspectos que se han reportado en la literatura como característicos de la restauración psicológica (Hartig y Staats, 2006; Kaplan, 1995). Los factores encontrados incluyeron los 17 reactivos con cargas $>.40$. Los niveles de consistencia interna, coeficiente alfa total 0.85, son adecuados (Nunnally y Bernstein, 1994). Es decir, se adaptó la escala de percepción de restauración ambiental de manera válida y confiable. La evaluación de la restauración psicológica es necesaria para conocer qué ambientes pueden ser restaurativos (Hartig, Korpela, Evans, y Gärling, 1997). Los ambientes de esta naturaleza son catalizadores de distintos procesos como cognitivos y físicos que son propios del ciclo vital de los individuos; son escenarios que pueden promover la renovación de los recursos físicos, psicológicos y sociales ante las demandas adaptativas que provienen de los escenarios sociofísicos, y por ello pueden promover la salud mental (Patrick y Capetola, 2011; Townsend, 2006).

En esta forma, la evaluación de la restauración es necesaria para conocer las características de los escenarios naturales como los jardines, que en sí mismos son restaurativos, pero con la especificación de sus visitantes puede incrementar sus efectos positivos en la renovación y equilibrio de los recursos físicos, psicológicos y sociales ante las demandas de lugares como la zona metropolitana de México (Bustos, Rincón y Flores, 2011).

Es importante señalar algunas limitaciones del instrumento, por ejemplo, se deberán revisar los reactivos de cada factor con 
la finalidad de: por un lado, mejorar su contenido e incorporar una mayor variedad de aspectos vinculados con restauración y, por otro, recuperar el factor de "Compatibilidad", cuyos reactivos carecieron de carga factorial adecuada en el estudio. También buscar el aumento en los niveles de confiabilidad por factor, sobre todo coherencia, si se intenta relacionar la escala obtenida con variables sociodemográficas y contextuales con la restauración psicológica. Esta situación demandará el incremento del tamaño de la muestra, manteniendo el equilibrio en el número de hombres y mujeres que responden a la escala, por lo que para futuros estudios se tendrá que ampliar la misma y posiblemente buscar replicar el estudio en diversas muestras de jóvenes y adultos.

Podemos concluir que los hallazgos obtenidos en el estudio sientan las bases para estudiar con una escala válida y confiable la restauración en jardines para población mexicana.

\section{Referencias}

Bustos, A.J.M., Rincón, L.G. y Flores, H.L.M. (2011). Exploración de las creencias sobre la escasez de agua en población de la Ciudad de México. Quaderns de Psicología, 13(1), 13-23.

Fitch, J. (1983). Bases empíricas de la dimensión estética. En H.M. Proshansky, W. Ittelson y L.G. Rivlin (Eds.), Psicología Ambiental. El hombre y su entorno social (113-123). México: Trillas.

Hartig, T., Korpela, K., Evans, G., y Gärling, T. (1997). A measure of Restorative Quality in Environments. Scandinavian Housing \& Planning Research, 14, 175-194.

Hartig, T. (2003). Restorative Environments. Journal of Environmental Psychology, 23, 103-107.

Hartig, T. y Staats, H. (2006). The Need of Psychological Restoration as a Determinant of Environmental Preferences. Journal of Environmental Psychology, 26, 215-226.

Kaplan, R. y Kaplan, S. (1989). The Experience of Nature: A Psychological Perspective. Nueva York: Cambridge University Press. 
Kaplan, S. (1995). The restorative Benefits of Nature: Toward an Integrative Framework. Journal of Environmental Psychology, 15, 169-182.

Martínez Soto, J. (2010). Impacto de la naturaleza urbana próxima. Un modelo de ecología social. (Tesis inédita de doctorado). México: unAm.

Nunnally, J.C. y Bernstein, I.H. (1994). Psychometric Theory. Nueva York: McGraw-Hill.

Patrick, R. y Capetola, T. (2011). It's Here! Are We Ready? Five Case Studies of Health Promotion Practices that Address Climate Change from within Victorian Health Care Settings. Health Promotion Journal of Australia, 22, 61-67.

Townsend, M. (2006). Natural Connections: Connecting with Nature is Really Good for Human Health and Wellbeing. VicHealth Letter, 26, 14-18.

Ulrich, R. (1983) Aesthetic and Affective Response to Natural Environment. En I. Altman y J. Wohlwil (Eds.). Human Behavior and Environment: Advances in Theory and Research (Vol. 6). Behavior and the Natural Environment (85-125). Nueva York: Plenum Press.

Van Den Berg, A., Koole, L., y Van Der Wulp, N. (2003). Environmental Preference and Restoration: How Are They Related? Journal of Environmental Psychology, 23, 135-146.

Van Den Berg, A. Hartig, T. y Staats, H. (2007). Preference for Nature in Urbanized Societies: Stress, Restoration, and the Pursuit of Sustainability. Journal of Social Issues, 63, 79-96. 
